\title{
Multiparametric MRI Versus Multiparametric US in the Detection of Prostate Cancer
}

\author{
FRANCESCO M. DRUDI ${ }^{1}$, VITO CANTISANI ${ }^{1}$, FLAVIA ANGELINI ${ }^{1}$, MAURO CICCARIELLO ${ }^{1}$, \\ DANIELA MESSINEO ${ }^{1}$, EVARISTO ETTORRE ${ }^{2}$, MAURO LIBERATORE ${ }^{1}$ and MICHELE SCIALPI ${ }^{3}$ \\ ${ }^{1}$ Department of Radiological, Oncological and Pathological anatomy Sciences, \\ Sapienza University of Rome, Rome, Italy; \\ ${ }^{2}$ Department of Cardiovascular, Respiratory, Nephrological, \\ Anaesthetic and Geriatric, Sapienza University of Rome, Rome, Italy; \\ ${ }^{3}$ Section of Diagnostic Imaging, Department of Surgical and Biomedical Sciences, \\ Santa Maria della Misericordia Hospital, University of Perugia, Perugia, Italy
}

\begin{abstract}
Background/Aim: The aim of the study was to compare the diagnostic accuracy of multiparametric magnetic resonance imaging (mpMRI), multiparametric ultrasound (mpUS) and US/MRI fusion imaging techniques in the detection of prostate cancer. Patients and Methods: A total of 82 patients with persistently high prostate specific antigen (PSA) levels after medical therapy were prospectively evaluated. All patients underwent digital rectal examination, mpMRI, mpUS and prostate biopsy. Results: Histological outcome was positive for prostate cancer ( $P C a)$ in 46/82 patients (56.1\%). MpMRI detected 54/82 lesions; histological analysis confirmed PCa in 44 lesions (sensitivity $91.3 \%$ and specificity $66.7 \%$ ). Ratio estimation with semiquantitative elastography, between lesions and the peripheral portion showed a higher sensitivity and specificity compared to strain ration (SR) evaluation between lesions and adenomas (sensitivity $84.8 \%$ vs. $78.3 \%$; specificity $66.6 \%$ vs. $61.1 \%)$. Quantitative analysis of contrastenhanced ultrasound (CEUS) showed $40.0 \%$ sensitivity and 97.2\% specificity. A total of 54 lesions detected by mpMRI and MRI/TRUS fusion targeted biopsy had a high number of positive samples (81.5\%). Conclusion: mpMRI is more accurate than mpUS which still remains a valuable technique used after MRI for prostate fusion-guided biopsy.
\end{abstract}

Prostate cancer $(\mathrm{PCa})$ is one of the most common cancers in men in the western world. The American Cancer Society's

Correspondence to: Francesco M. Drudi, University Sapienza of Rome, Rome, Italy. Tel: +39 0649978439, e-mail: francescom.drudi@ uniroma1.it

Key Words: Multiparametric MRI, multiparametric US, prostate cancer, diagnostic imaging. estimates for prostate cancer in the United States in 2019 indicate that there are about 174,650 new cases and about 31,620 deaths related to this disease (1).

Multiparametric magnetic resonance imaging (mpMRI) of the prostate - including morphological T2-weighted (T2w), and functional imaging such as diffusion-weighted imaging (DWI), dynamic contrast-enhanced imaging (DCE), and MRI spectroscopy (MRS) $(2,3)$, provide accurate detection of $\mathrm{PCa}$ and are recommended in patients with persistent clinical suspicion of PCa despite prior negative biopsies (4).

However, the technical heterogeneity of the methods, the lack of standards for image interpretation and the management of category 3 lesions are the main drawbacks that prevent a wider acceptance of mpMRI in clinical practice (4).

Currently, bi-parametric MRI (bpMRI) (T2w and DWI) yields similar results to mpMRI in terms of detection and in guiding MRI/US fusion targeted biopsy of suspected PCa, and has the advantages of reducing costs and acquisition time, and eliminating the risks of gadolinium contrast basedagent $(5,6)$.

Multiparametric ultrasound (mpUS) describes a combination of different US examinations (7) including grayscale US, color Doppler (CDUS) and power Doppler (PDUS), transrectal elastography (TRES) and contrastenhanced ultrasound (CEUS).

Grayscale US is a more useful first-line imaging method than digital rectal examination (DRE) with high sensitivity and specificity $(8,9)$. However, when DRE is negative and prostate-specific antigen (PSA) levels are normal, only a small number of malignancies are found at grayscale US (10). Although CDUS and PDUS do not increase sensitivity in the diagnosis of PCa, these methods allow detection of neoplastic foci not visible on grayscale US by showing abnormal vascularity $(3,11)$. 
TRES is a relatively new investigative tool that outlines possible stiffness of prostate tissues (8). TRES has given rise to the development of various imaging techniques that evaluate tissue properties to create an image based on their deformation during mechanical compression or an elastogram reflecting the different velocities of shear wave propagation using pulse echo US (8).

To improve results of US guided biopsies, CEUS has been developed to detect changes in blood flow as this improves detection of malignancies, including PCa (12).

Transrectal US (TRUS)-guided prostate biopsy is considered the gold standard for tissue sampling and diagnosis of PCa. According to the current European Association of Urology (EAU) guidelines, random biopsy should include twelve cores thereby leading to a detection rate ranging between $24 \%$ and $44 \%$. Also, mpMRI is a widely used technique for PCa detection and management and for guiding MRI/US fusion-targeted biopsy, thus offering a feasible alternative to TRUS.

However, a combination of US and MRI images may offer a feasible alternative to TRUS. Different US/MRI fusion imaging techniques are employed to identify the index lesion and guide transrectal or transperineal biopsy procedures. Fusion imaging integrates the data obtained at previously performed mpMRI with real-time US imaging to guide the biopsy needle to the target identified at MRI.

The aim of this study was to compare the diagnostic accuracy of mpMRI and mpUS in the detection of PCa.

\section{Materials and Methods}

Patient population and study design. We prospectively evaluated 82 consecutive patients (median age 68.8 years, range $=51-85$ years) who underwent mpMRI and mpUS form December 2015 to June 2017. The study was approved by the local Ethics Committee and all patients provided written informed consent.

Inclusion criteria were: (a) Total PSA level $>4 \mathrm{ng} / \mathrm{ml}$; (b) PSA density $>0.15 \mathrm{ng} / \mathrm{ml}^{2}$, (c) PSA velocity $>0.75 \mathrm{ng} / \mathrm{ml} / \mathrm{y}$; (d) Free/total PSA ratio $<0.10$ with total PSA level between 4 and $10 \mathrm{ng} / \mathrm{ml}$; (e) Persistently high PSA values after medical therapy.

All patients had to meet all five criteria and all underwent DRE, mpMRI and mpUS and subsequent prostate biopsy. All patients had to meet all five criteria and all underwent DRE, mpMRI and mpUS as well as prostate biopsy, according to simplified Prostate Imaging Reporting and Data System (S-PIRADS) (6).

MpMRI. Pelvic MRI focused on the prostate gland was performed using a 3 Tesla (3T) magnet (Discovery MAGNETOM Verio Siemens Medical Solutions) equipped with a phased-array coil and an endorectal coil.

MRI protocol included the following sequences: a) T2w turbo spin-echo sequences (repetition time (TR) 4,500 msec; echo time (TE) $110 \mathrm{msec}$; thickness $3 \mathrm{~mm}$; matrix $512 \times 352$ ) in axial, sagittal, and coronal planes; b) DWI sequences: slice thickness $3 \mathrm{~mm}$; TR 3,100 msec; TE $102 \mathrm{msec}$; exponential b values $0,500,1,000$ and $2,000 \mathrm{~s} / \mathrm{mm}^{2} ; \mathrm{c}$ ) DCE MRI images were obtained using gradient-echo
T1-weighted (T1w) sequences in the axial plane (TR 3 msec; TE 2 msec; thickness $3 \mathrm{~mm}$; time resolution $12 \mathrm{sections} / 3 \mathrm{sec}$; and matrix $320 \times 192$ ) after i.v. of gadolinium-based contrast material of 0.1 $\mathrm{ml} / \mathrm{Kg}$ of Gadobutrol (Gadovist, Bayer Schering Pharma AG, Berlin, Germany). At each mpMRI sequence (T2w, DWI and DCE) a score was assigned according to the PI-RADS version 2 (PI-RADS v2) (13). Category 3 lesions were managed according to S-PIRADS (6).

MpUS. MpUS examinations were performed using the following US equipment: Hitachi Ascendus (Hitachi Medical, Tokyo, Japan), Toshiba Aplio 500 (Toshiba, Osaka, Japan), Esaote Twice (Esaote, Genoa, Italy) and an endocavitary biplane or end-fire multifrequency transducer (4-8 MHz).

All patients underwent TRUS, CDUS, PDUS, TRES and CEUS in the same US session.

Grayscale US was carried out to identify the presence of a focal lesion in the peripheral portion of the prostate. CDUS and PDUS were carried out to detect possible vascular flow within the lesion. Elastography was carried out to obtain a color map of the prostate. The US operator applied manual compression and decompression using a transrectal probe while observing the degree of compression on the US monitor. Qualitative evaluation was carried out by dividing the tissues into five color patterns from purple-blue (max stiffness) to red (max elasticity).

For a quantitative analysis, three regions of interest (ROIs) were selected at the base, middle third and apex of the prostate gland in the right and left peripheral zone (PZ) and in the central portion/adenoma. Strain ratios (SRs) were calculated between the two peripheral portions and between the right and left peripheral portions and the central portion.

CEUS was carried out after intravenous administration of $4.8 \mathrm{ml}$ contrast medium (SonoVue, Bracco, Milan, Italy) followed by $5 \mathrm{ml}$ saline solution, using a low mechanical index (MI) (range $=0.06-$ 0.1 ). The spread of contrast medium was displayed in real-time fashion evidencing the lesion and healthy parenchyma (duration approximately $2.30 \mathrm{~min}$ ).

The obtained video clips were analyzed qualitatively defining the type of enhancement as: absent (score 0), modest (score 1) or intense (score 2). Quantitative analysis included creation of time/intensity curves using dedicated software.

MRI/US fusion-guided prostate biopsy. A rigid transformation system was used (Virtual Navigator, Esaote, Genoa, Italy). This system provides a geometrical transformation that preserves all distances and straightness of lines as well as translation and rotational variations of the images. Virtual Navigator advanced system allows real-time visualization of enhanced US images due to correlation with MRI gold-standard images. The combination of US and MRI reference methods permits fusion of data thereby increasing the ability to assess lesion morphology particularly in difficult-to-scan patients.

Transperineal biopsy of the prostate (using a 16G needle) included 14 or more cores: 2 target biopsies and 6 random biopsies taken from each portion of the peripheral prostate wall divided into sextants. One core was taken on each side of the medial and lateral lobe of the glandular base ( 2 cores), one core was taken from the medial and lateral lobe of the middle third portion of the gland ( 2 cores), and one sample was taken from the apex ( 2 cores) for a total of 12 biopsies.

All biopsy samples were examined by the same pathologist with longstanding experience in urogenital diseases. Samples were 
Table I. Summary of results obtained in patients not affected by $P C a$ $\left(N^{\circ}=36 / 82\right)$.

\begin{tabular}{lccc}
\hline $\begin{array}{l}\text { Histological } \\
\text { results }\end{array}$ & $\begin{array}{c}\mathrm{N}^{\circ} \\
\text { Patients }\end{array}$ & $\begin{array}{c}\text { PSA }(\mathrm{ng} / \mathrm{ml}) \\
\text { range }\end{array}$ & $\begin{array}{c}\text { Maximum diameter }(\mathrm{cm}) \\
\text { range }\end{array}$ \\
\hline Normal & 13 & $2.89-9.67$ & $<1$ \\
LGPIN & 10 & $4.60-13.00$ & $1.1-2.0$ \\
HGPIN & 7 & $0.94-8.02$ & $2.1-3.0$ \\
ASAP & 2 & $4.70-15.00$ & $>3.0$ \\
PIA & 4 & $0.94-5.69$ & $2.6-3.4$ \\
\hline
\end{tabular}

LGPIN: Low-grade prostatic intraepithelial neoplasia; HGPIN highgrade prostatic intraepithelial neoplasia; ASAP: atypical small acinar proliferation; PIA: proliferative inflammatory atrophy.

classified according to the Gleason classification system: a) free from cancer lesion; b) precancerous lesion: low-grade prostatic intraepithelial neoplasia (LGPIN), high-grade prostatic intraepithelial neoplasia (HGPIN), atypical small acinar proliferation (ASAP), proliferative inflammatory atrophy (PIA); c) PCa.

Images were evaluated by two radiologists with longstanding experience in genitourinary radiology.

Statistical analysis. Sensitivity, specificity, diagnostic accuracy and positive and negative predictive values (PPV and NPV, respectively) were calculated. Furthermore, Bayesian analysis was carried out by correlating PPV and NPV (post-test probability) with the "a priori" probability of having the disease (pre-test probability). This analysis indicates which test provides sensitivity and specificity values compatible with the pre-test probability (prevalence of disease) of each patient. This occurs also when the "a priori" probability of a test is represented by the posterior probability found with another test.

Reliability of the diagnostic procedures was calculated on the same sample of patients and therefore the prevalence of disease was the same. This allowed the comparison not only of sensitivity and specificity, which do not depend on the prevalence of disease, but also of the predictive values, which depend on the specificity. The predictive value of a test can also be interpreted in probabilistic terms. Prevalence of disease is calculated on the basis of the number of affected patients included in the sample, but in the single patient the prevalence identifies the "a priori" or "pre-test" probability. Such probability does not necessarily correspond to the overall prevalence of disease in the population, as this parameter may vary according to the condition of the single patient. In turn, the predictive values calculated identify "a posterior" or "post-test" probability of malignancy.

\section{Results}

MpMRI, US and biopsy procedures were tolerated in all patients. No significant adverse reactions to contrast agents occurred.

Histological outcome was positive for $\mathrm{PCa}$ in $46 / 82$ patients $(56.1 \%)$ whereas $36 / 82$ patients were negative for malignancy or presented mild atypia (Table I). Gleason scores and PSA values in the 46 cases of PCa are reported in Table II and Table
Table II. Summary of Gleason score values obtained in patients affected by $P C a\left(N^{\circ}=46 / 82\right)$.

\begin{tabular}{lc}
\hline Gleason score & $\mathrm{N}^{\circ}$ Patients $(\%)$ \\
\hline $6(3+3)$ & $22(26.8)$ \\
$7(3+4)$ & $14(17.0)$ \\
$8(4+4)$ & $6(7.3)$ \\
$9(4+5)$ & $4(4.8)$ \\
\hline
\end{tabular}

Table III. Summary of PSA values obtained in patients affected by PCa $\left(N^{\circ}=46 / 82\right)$

\begin{tabular}{lc}
\hline PSA $(\mathrm{ng} / \mathrm{ml})$ & $\mathrm{N}^{\circ}$ Patients $(\%)$ \\
\hline $4.0-5.9$ & $13(28.3)$ \\
$6.0-7.9$ & $15(32.6)$ \\
$8-10$ & $14(30.4)$ \\
$>10$ & $4(8.7)$ \\
\hline
\end{tabular}

III, respectively. All 82 patients underwent mpMRI, which identified 54 lesions classified as follows: PI-RADS $5(n=16)$, PI-RADS $4(\mathrm{n}=24)$ and PI-RADS $3(\mathrm{n}=14)$.

Histological analysis confirmed $\mathrm{PCa}$ in 44 lesions whereas 10 lesions assigned to PI-RADS score 3 were false positive. In the detection of suspicious PCa, MpMRI yielded similar sensitivity compared to bpMRI (T2w and DWI sequences); DCE increased specificity of mpMR in 4 patients.

Grayscale TRUS detected 40/82 lesions. CDUS/PDUS results were similar to that of grayscale TRUS. Elastography was carried out for qualitative and quantitative assessment of the prostate tissues. Qualitative analysis identified 52/82 lesions of which 36 were positive for $\mathrm{PCa}$, whereas 16 were false positive.

Quantitative analysis/strain ratio (SR) between the central portion and peripheral zone identified 61/82 lesions of which 36 were positive for PCa (Table IV). SR between the two peripheral zones identified 64/82 lesions of which 39 were positive for PCa (Table V).

Qualitative analysis of CEUS identified 28/82 lesions: 12 presented a significant increase in vascularity compared to the surrounding peripheral zone (score 2), whereas 16 presented moderate increase in vascularity (score 1). Histological analysis showed that 8 of the 12 lesions assigned score 2 were positive for PCa.

Of the 16 patients assigned to score 1 , only one patient was positive for PCa.

Quantitative analysis of CEUS identified 18/82 lesions which were characterized by suspicious curves with a quick wash-in followed by a fast wash-out; of these 18 lesions, 14 

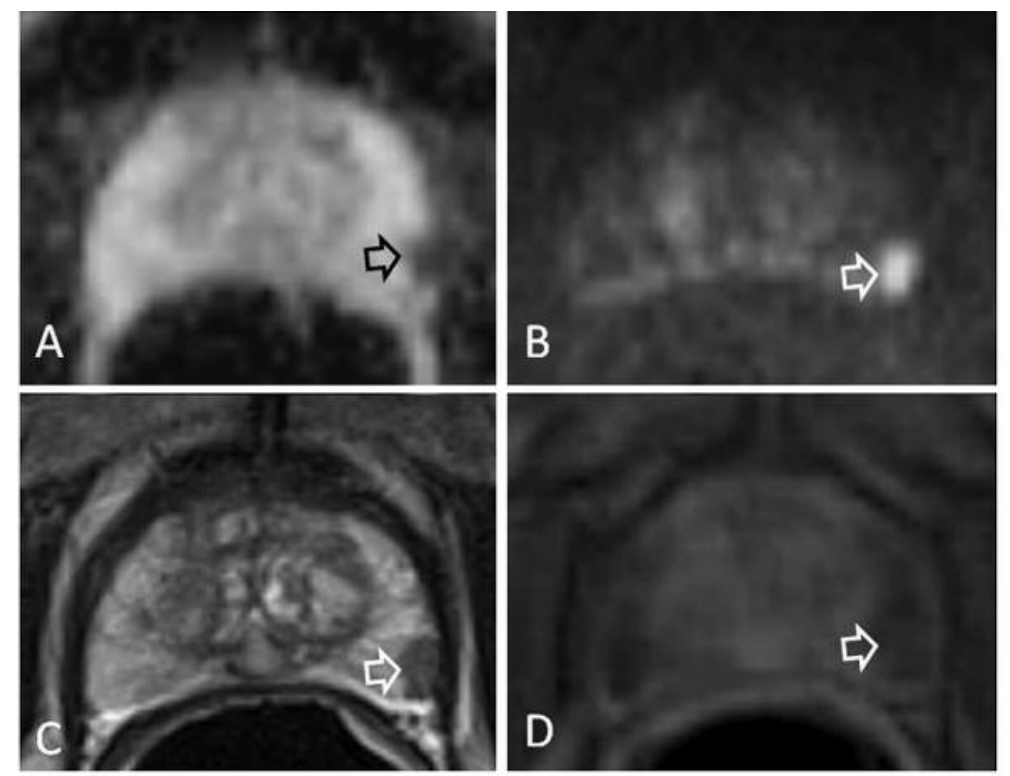

Figure 1. Multiparametric MR imaging of the prostate in a 56-year-old patient with elevated PSA, shows in the mid of the gland in the left peripheral zone a homogeneous marked hypointense area on ADC (arrow in A), hyperintense on high b-value diffusion-weighted imaging (arrow in B), and hypointense on T2-weighted image (arrow in C) without contrast enhancement on dynamic-contrast enhanced imaging (arrow in D).

Table IV. Reliability of used imaging modalities in patients with suspected prostatic cancer.

\begin{tabular}{|c|c|c|c|c|c|c|c|c|c|}
\hline & VP & VN & FP & FN & $\mathrm{Se}(\%)$ & $\mathrm{Sp}(\%)$ & $\operatorname{Acc}(\%)$ & PPV & NPV \\
\hline TRUS & 26 & 22 & 14 & 20 & 56.5 & 61.1 & 58.5 & 65.0 & 52.4 \\
\hline CDUS & 20 & 14 & 22 & 26 & 43.5 & 38.9 & 41.5 & 47.6 & 35.0 \\
\hline QUALITATIVE CEUS & 8 & 32 & 4 & 38 & 17.4 & 88.9 & 48.8 & 66.7 & 45.7 \\
\hline QUANTITATIVE CEUS & 17 & 35 & 1 & 29 & 40.0 & 97.2 & 63.4 & 94.4 & 54.7 \\
\hline QUALITATIVE TRES & 32 & 16 & 20 & 14 & 69.6 & 44.4 & 58.5 & 61.5 & 53.3 \\
\hline TRES S/R A/L & 36 & 22 & 14 & 10 & 78.3 & 61.1 & 70.7 & 72.0 & 68.7 \\
\hline TRES S/R PZ/L & 39 & 24 & 12 & 7 & 84.8 & 66.6 & 76.8 & 76.5 & 77.4 \\
\hline mp MRI & 44 & 32 & 4 & 2 & 95.6 & 88.9 & 92.7 & 91.7 & 94.1 \\
\hline bp MRI & 44 & 28 & 6 & 4 & 91.7 & 82.3 & 87.8 & 88.0 & 87.5 \\
\hline
\end{tabular}

Table V. SR periferal zone/lesion.

GLEASON 6: 42.4/16 lesions $=2.65$

GLEASON 7: $14.56 / 13$ lesions $=1.12$

GLEASON 8-10: 18.1/10 lesions=1.09

were confirmed positive for PCa at histological analysis. The prevalence of disease was $56 \%$ (i.e. the proportion of patients affected by the disease). TRES SR/peripheral zone and mpMRI provided more accurate values than the other diagnostic procedures. The reliability of each procedure is reported in Table VI.
Table VI. Media SR adenoma/lesion.

GLEASON 6: 21.15/15 lesions $=1.41$

GLEASON 7: 20.04/12 lesions $=1.67$

GLEASON 8-10: 22.5/9 lesions $=2.50$ 


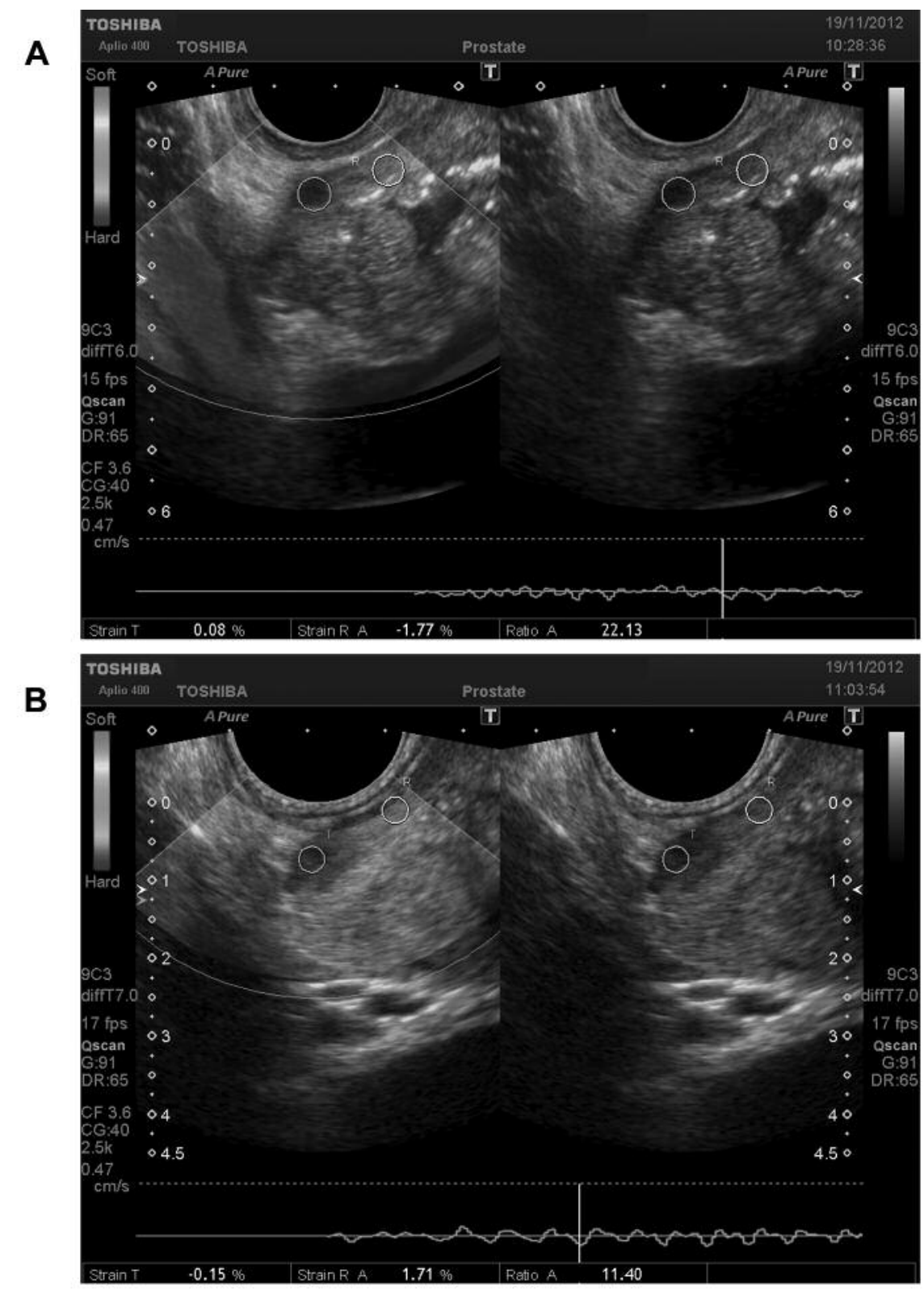

Figure 2. Elastosonography of PCa. A: Strain and Elastography Ratio estimation between lesions and the peripheral portion. B: Same procedure in a different patient.

with a US for fusion-guided biopsy by a skilled radiologist with experience.

Ahmed et al. have found that the sensitivity of mpMRI in clinically significant cancer is approximately $93 \%$, with a negative predictive value of $89 \%$, specificity of $41 \%$ and a positive predictive value of $51 \%$ (14).

Turkbey et al. have shown that the addition of spectroscopy to conventional $3 \mathrm{~T}$ sequences, improves the predictive value of mpMRI-based PCa detection $(8,15)$.
Grayscale US examination is useful thanks to the easy accessibility and the possibility to take biopsies in real time during the examination, but is not accurate in the diagnosis of PCa. In a study reported by Pallwein et al., which correlated US results to radical prostatectomy specimens, elastography detected $88 \%$ of cancer foci, and elastography guided biopsies achieved a 2.9-fold higher detection rate than random biopsies in a group of 230 patients (16). 

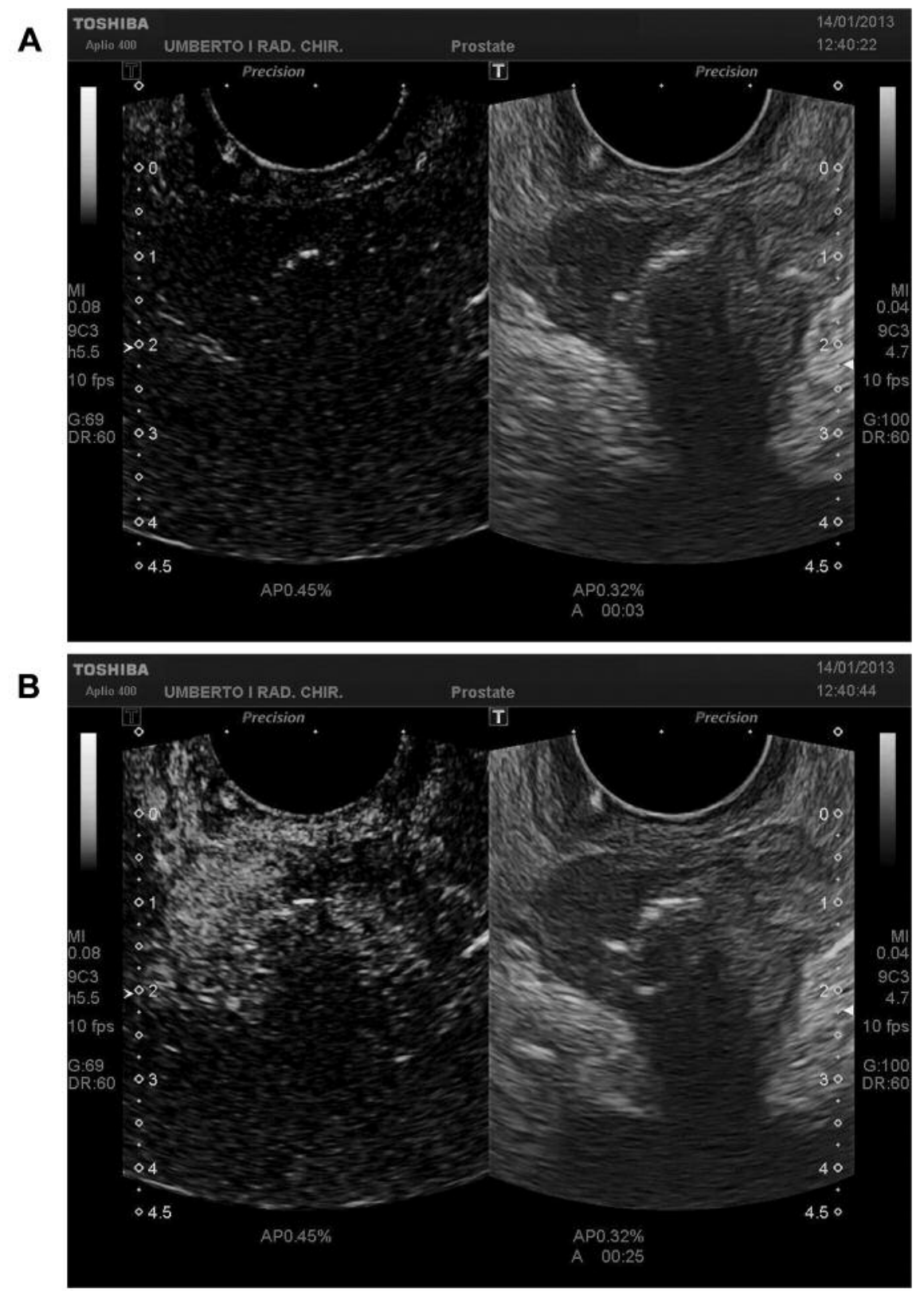

Figure 3. Continued

In 2012, Zhang et al. conducted a meta-analysis of seven studies that evaluated the performance of diagnostic elastography with radical prostatectomy specimens as standard (17). In the combined population of $508 \mathrm{men}$, the pooled sensitivity and specificity were $72 \%$ and $76 \%$, respectively.

Aigner et al. have demonstrated similar sensitivity and NPV for strain RTE and T2-weighted MRI in an analysis of peripheral zone lesions in 33 patients (17).

In a study by Pelzer et al., 50 patients with biopsy-proven cancer underwent strain RTE and mpMRI to detect PCa. The results were analyzed retrospectively and correlated with RP specimens. Sensitivity of strain RTE was higher than that of mpMRI (92\% vs. 84\%), but the authors commented that the MRI findings may have been confounded by prior biopsies that cause hemorrhaging artifacts on MRI (18).

In a meta-analysis of 16 studies including 2624 patients, $\mathrm{Li}$ et al. have found a pooled sensitivity and specificity of $70 \%$ and $74 \%$, respectively, in PCa detection using CEUS imaging (8). MRI/US fusion biopsy has been shown to be reliable and to lead to increased detection rates of PCa (19-22). Tewes et al. (19) have shown that targeted biopsy in lesions classified 


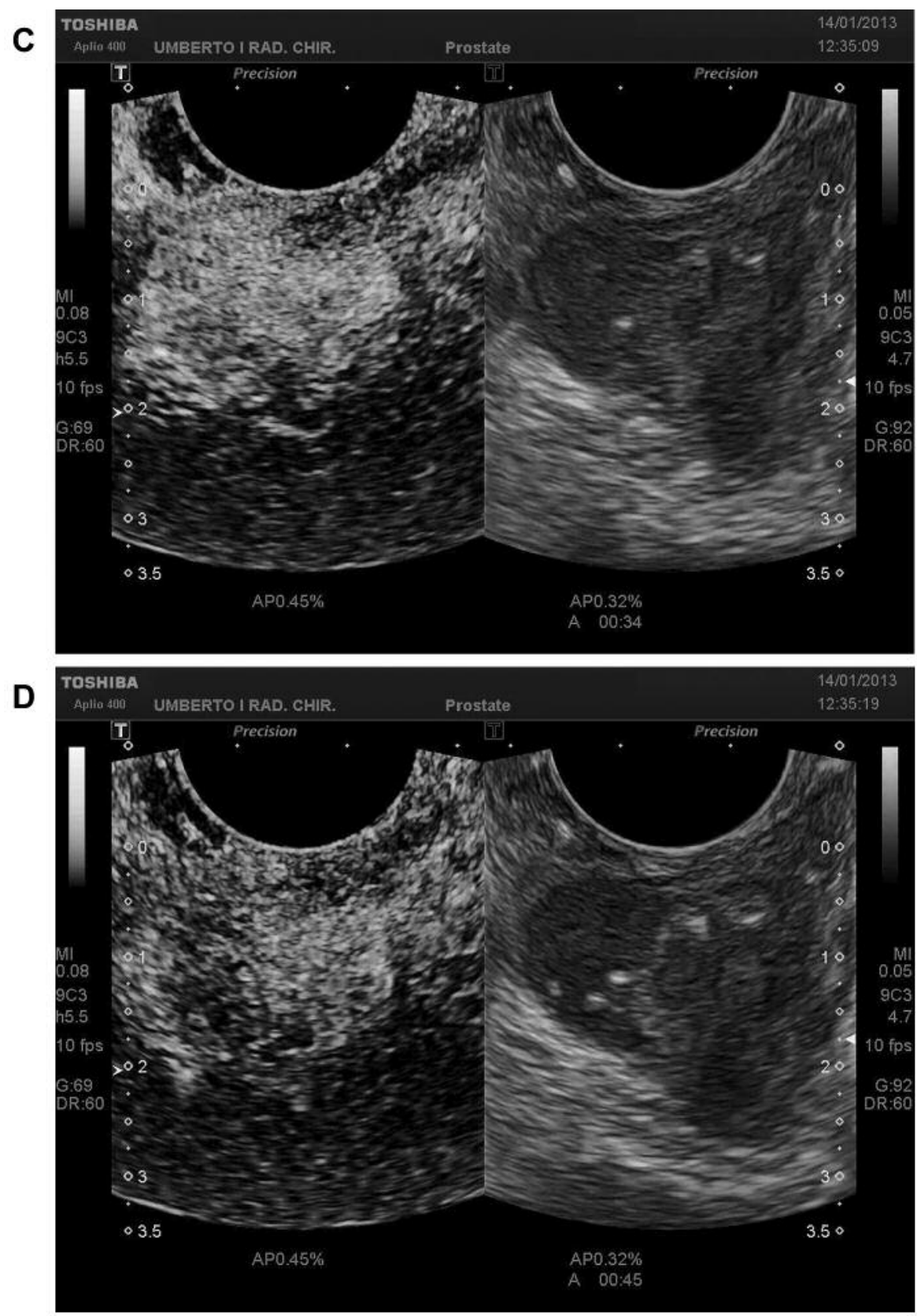

Figure 3. CEUS in evaluation of PCa. A: US imaging immediately after contrast injection shows no contrast enhancement in the gland. B: after 25 sec, a hyper-enhanced area is visible in the suspected neoplastic lesion. C: After 34 sec the lesion appears nearly contrast-filled. In the deep portion of the gland there is also an enhanced area which was not clearly depicted at the previous US gray scale examination. D: After 45 sec wash-out starts in the lesion and the gland is full of contrast agent.

PI-RADS $\geq 4$ achieved sensitivity $84 \%$, specificity $85 \%$ and NPV $92 \%$. In a systematic review reported by Valerio et al. in 2015 (20), the authors stated that an increased number of histologically confirmed tumors were found using MRI/US fusion guided biopsy and that $9.1 \%$ of cancers would have been missed at a systematic biopsy. Kongyuy et al. (22) have highlighted the additional benefit of employing MRI/US fusion guided biopsy in patients with negative systematic TRUS-guided biopsy as an alternative to 20-core biopsy and in patients undergoing active surveillance for PCa.

In our study mpMRI, mpUS and fusion imaging were compared to biopsy, and to obtain useful statistical values, 


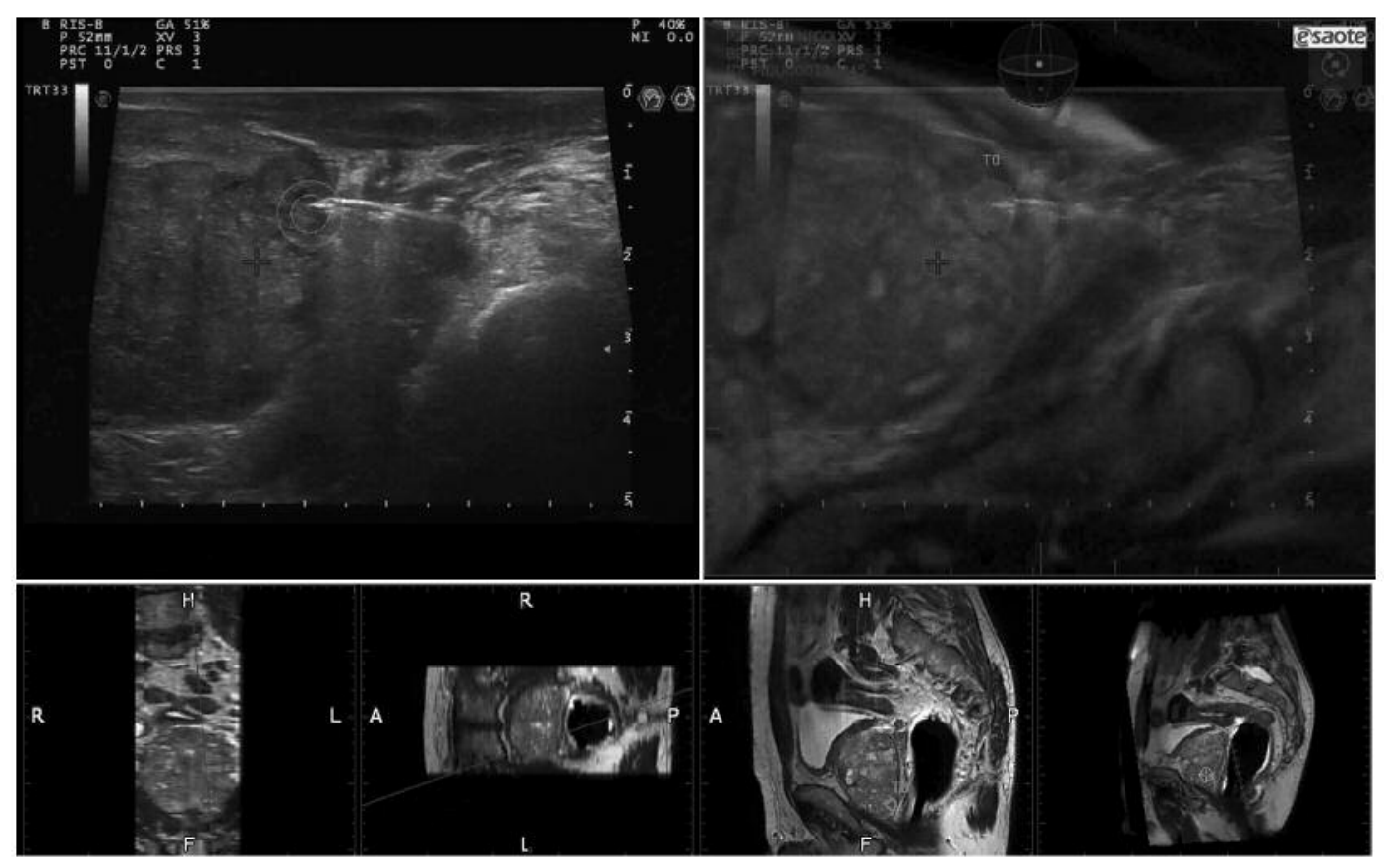

Figure 4. Fusion imaging for target biopsy of small lesion.

all techniques were compared to each other. "Pre-test" probability of having the disease was quite high (56\%) in the studied patient population. As can be seen, only TRES and MRI produce a significant increase in PPV over "pre-test" probability, but MRI achieved a higher NPV, which was particularly useful in the present series characterized by a high prevalence of disease.

Diagnosis of PCa is based on histological analysis and requires confirmation by biopsy. In our study, inclusion criteria allowed selection of a patient population in which the probability of cancer diagnosis was high. MpMRI detected $54 / 82$ lesions and 14 lesions with a volume $>0.5 \mathrm{ml}$ were categorized as PI-RADS category 3 identifying 4 out of 14 $(28.5 \%)$ as PCa and 10 out of $14(71.5 \%)$ as benign lesions (false positive). These results are in accordance with those reported in the literature (6).

However, according to our study, mpMRI is still the best diagnostic technique, achieving sensitivity $91.3 \%$ and specificity $66.7 \%$ (Figure 1).

Qualitative elastography achieved a low sensitivity $(69.6 \%)$ and specificity $(44.4 \%)$. This is probably due to compression of the peripheral zone by the adenoma, which alters tissue stiffness reading during the computation of the elastographic map. Semiquantitative elastography was performed to evaluate SRs between the lesion and the central adenoma and between the lesion and the peripheral zone of the gland. SR between the adenoma and the lesion tends to decrease as Gleason score increases. This probably occurs because the central adenoma shows a more homogeneous stiffness except in the presence of granulomatous prostatitis and central portion carcinoma which might change the ratio, but this occurs less frequently. Ratio estimation between lesions and the peripheral portion showed higher sensitivity and specificity compared to SR evaluation between lesions and adenoma (sensitivity $84.8 \%$ vs. $78.3 \%$; specificity $66.6 \%$ vs. $61.1 \%$ ). However, correlation with Gleason score values revealed that the difference in SR was greater (mean 2.65) in lesions assigned low scores (Gleason $\leq 6$ ) and that SR decreased with increasing Gleason scores reaching minimum at Gleason scores 8-10. This may be also due to neoplastic infiltration of the adjacent peripheral zone (Figure 2A and B).

Qualitative analysis of CEUS (28/82 lesions) showed low sensitivity and high specificity $(17.4 \%$ and $88.9 \%$, respectively). In fact, 12/28 lesions presented marked hyper enhancement with clear distinction between the lesion and the surrounding normal parenchyma (Figure 3A-D).

Quantitative analysis of CEUS showed sensitivity $40.0 \%$ and specificity $97.2 \%$. However, only 18/82 lesions showed the characteristic quick wash-in and rapid wash-out patterns; 14 of these 18 lesions were malignant. This low percentage of hypervascular lesions indicates that CEUS is not a very valid tool in PCa detection.

It is well known that fusion imaging biopsy reduces the number of random biopsy samples. Our study confirms this 
assumption: 54 lesions detected by mpMRI and MRI/TRUS fusion targeted biopsy presented a high number of positive samples $(81.5 \%)$ (Figure 4).

Our results demonstrated that mpMRI yields a sensitivity similar to that of bpMRI in the detection of suspicious PCa; both methods are more accurate than mpUS which still remains a valuable technique to be used after MRI for prostate fusion-guided biopsy.

In conclusion in clinical practice, mpMRI and bpMRI combined with TRUS/TRES prostate fusion-guided biopsy are accurate and useful methods for detecting clinically significant PCa.

\section{Conflicts of Interest}

The Authors have no conflicts of interest to disclose regarding this study.

\section{Authors' Contributions}

FMD: Supervised and carried out examinations and wrote the manuscript; VC: carried out examinations; FA: collected references; MC: carried out examinations and biopsies; DM: Statistical analyses; EE: assisted in the drafting of the manuscript; ML: carried out and supervised statistical analyses; MS: supervised the text.

\section{References}

1 Siegel RL, Miller KD and Jemal A: Cancer statistics, 2019. CA Cancer J Clin 69(1): 7-34, 2019. PMID: 30620402. DOI: $10.3322 /$ caac. 21551

2 Rooij M, Hamoen EH, Futterer JJ, Barentsz JO and Rovers MM: Accuracy of multiparametric MRI for prostate cancer detection: a metaanalysis. AJR Am J Roentgenol 202: 343-345, 2014. PMID: 24450675. DOI: 10.2214/AJR.13.11046

3 Tangel MR and Rastinehad AR: Advances in prostate cancer imaging. F1000Res 7: pii: F1000, 2018. PMID: 30467517. DOI: 10.12688/f1000research.14498.1

4 Mottet N, Bastian PJ, Bellmunt J, van den Bergh RCN, Bolla M,van Casteren NJ, Cornford P, Joniau S, Mason MD, Matveev $\mathrm{V}$, van der Kwast $\mathrm{TH}$, van der Poel $\mathrm{H}$, Rouvière $\mathrm{O}$ and Wiegel T: Guidelines on Prostate Cancer. Eur Urol 65(1): 124-137, 2014. PMID: 24207135. DOI: 10.1016/j.eururo.2013.09.046

5 Scialpi M, D'Andrea A, Martorana E, Malaspina CM, Aisa MC, Napoletano M, Orlandi E, Rondoni V, Scialpi P, Pacchiarini D, Palladino D, Dragone M, Di Renzo G, Simeone A, Bianchi G, and Brunese L: Biparametric MRI of the prostate. Turk J Urol 43(4): 401-409, 2017. PMID: 29201499. DOI: 10.5152/ tud.2017.06978

6 Scialpi M, Aisa MC, D’Andrea A and Martorana E: Simplified prostate imaging reporting and data system for biparametric prostate MRI: A Proposal. AJR Am J Roentgenol 211(2): 379382, 2018. PMID: 29894218. DOI: 10.2214/AJR.17.19014

7 Sidhu PS: Multiparametric Ultrasound (MPUS) Imaging: Terminology describing the many aspects of ultrasonography. Ultraschall Med 36(4): 315-317, 2015. PMID: 26241118. DOI: $10.1055 / \mathrm{s}-0035-1553381$
8 Sarkar S and Das S: A review of imaging methods for prostate cancer detection. Biomed Eng Comput Biol 7: 1-15, 2016. PMID: 26966397. DOI: 10.4137/BECB.S34255

9 Otero JR, Gomez BG, Campos Juanatey F and Touijer KA: Prostate cancer biomarkers: an update. Urol Oncol 32(3): 252260, 2014. PMID: 24495450. DOI: 10.1016/j.urolonc. 2013.09.017

10 Cary $\mathrm{KC}$ and Cooperberg MR: Biomarkers in prostate cancer surveillance and screening: past, present, and future. Ther Adv Urol 5(6): 318-329, 2014. PMID: 24294290. DOI: 10.1177/ 1756287213495915

11 Tang J, Li S, Li J, Luo Y, Xu J, Zhang Y Li X, Shi H and Zhang G: Correlation between prostate cancer grade and vascularity on colour Doppler imaging: preliminary findings. J Clin Ultrasound 31: 61-68, 2003. PMID: 12539246. DOI: 10.1002/jcu.10139

12 Drudi FM, Di Candio G, Di Leo N, Malpassini F, Gnecchi M, Cantisani V, Iori $\mathrm{F}$ and Liberatore $\mathrm{M}$ : Contrast-enhanced ultrasonography in the diagnosis of upper urinary tract urothelial cell carcinoma: a preliminary study. Ultraschall Med 34(1): 30-37, 2013. PMID: 23165789. DOI: 10.1055/s-00321325548

13 Rosenkrantz AB, Oto A, Turkbey B and Westphale AC: Prostate Imaging Reporting and Data System (PI-RADS), Version 2: A Critical Look. AJR Am J Roentgenol 206: 1179-1183, 2016. PMID: 26913638. DOI: 10.2214/AJR.15.15765

14 Ahmed HU, El-Shater Bosaily A, Brown LC, Rhian Gabe R, Kaplan R, Parmar MK, Collaco-Moraes Y, Ward K, Hindley RG, Freeman A, Kirkham AP, Oldroyd R, Parker C, Emberton M and the PROMIS study group: Diagnostic accuracy of multiparametric MRI and TRUS biopsy in prostate cancer (PROMIS): A paired validating confirmatory study. Lancet 389(10071): 815822, 2017. PMID: 28110982. DOI: 10.1016/S0140-6736(16) 32401-1

15 Scialpi M, Scialpi P, Aisa MC, Martorana E and D'Andrea A: Simplified PI-RADS with biparametric MRI: A practical approach to improve Management of PI-RADS version 2 category 3 lesions. Radiology 289(3): 882-883, 2018. PMID: 30398444. DOI: $10.1148 /$ radiol.2018182092

16 Pallwein L, Mitterberger M, Pelzer A, Pallwein L, Mitterberger $\mathrm{M}$ and Pelzer A: Ultrasound of prostate cancer: recent advances. Eur Radiol 18(4): 707-15, 2008. PMID: 17938936. DOI: 10.1007/s00330-007-0779-7

17 Zhang B, Ma X, Zhan W, Zhu F, Li M, Huang J, Li Y, Xue L, Liu L and Wei Y: Real-time elastography in the diagnosis of patients suspected of having prostate cancer: a meta-analysis. Ultrasound Med Biol 40(7): 1400-1407, 2014. PMID: 24785435. DOI: $10.1016 /$ j.ultrasmedbio.2014.02.020

18 Aigner F, Pallwein L, Schocke M, Lebovici A, Junker D, Schäfer G, Mikuz G, Pedross F, Horninger W, Jaschke W, Halpern EJ and Frauscher F: Comparison of real-time sonoelastography with T2-weighted endorectal magnetic resonance imaging for prostate cancer detection. J Ultrasound Med 30(5): 643-649, 2011. PMID: 21527612.

19 Tewes S, Hueper K, Hartung D, Imkamp F, Herrmann TR, Weidemann J, Renckly S, Kuczyk MA, Wacker F and Peters I: Targeted MRI/TRUS fusion-guided biopsy in men with previous prostate biopsies using a novel registration software and multiparametric MRI PI-RADS scores: first results. World J Urol 33: 1707-1714, 2015. PMID: 25774003. DOI: 10.1007/s00345015-1525-4 
20 Valerio M, Donaldson I, Emberton M, Hadaschik BA, Marks LS, Mozer P, Rastinehad AR and Ahmed HU: Detection of clinically significant prostate cancer using magnetic resonance imaging-ultrasound fusion targeted biopsy: A systematic review. European urology 68(1): 8-19, 2015. PMID: 25454618. DOI: $10.1016 /$ j.eururo.2014.10.026

21 Maxeiner A, Stephan C, Durmus T, Slowinski T, Cash H and Fischer T: Added value of multiparametric ultrasonography in magnetic resonance imaging and ultrasonography fusion- guided biopsy of the prostate in patients with suspicion for prostate cancer. Urology 86: 108-114, 2015. PMID: 26142593. DOI: 10.1016/j.urology.2015.01.055
22 Kongnyuy M, George AK, Rastinehad AM and Pinto PA: Magnetic resonance imaging-ultrasound fusion-guided prostate biopsy: review of technology, techniques, and outcomes. Curr Urol Rep 17(4): 32, 2016. PMID: 26902626. DOI: 10.1007/s1 1934-016-0589-z

Received February 5, 2019

Revised May 14, 2019

Accepted May 20, 2019 